\title{
On the Uniqueness of Certain Type of Shift Polynomial Sharing a Small Function
}

\author{
Sujoy Majumder
}

\begin{abstract}
The purpose of the paper is to study the uniqueness problems of certain type of difference polynomial sharing a small function. We point out and rectify some gaps in the proof of the main results in [8]. In addition to this we obtain our main result as a corrected and generalized version of [8] in a more compact way which in turn improve a number of earlier results.
\end{abstract}

AMS Subject Classification (2000). 30D35

Keywords. Meromorphic function, difference polynomial, uniqueness

\section{Introduction, definitions and results}

In this paper by meromorphic functions we shall always mean meromorphic functions in the complex plane.

We adopt the standard notations of value distribution theory (see [5]). For a non-constant meromorphic function $f$, we denote by $T(r, f)$ the Nevanlinna characteristic of $f$ and by $S(r, f)$ any quantity satisfying $S(r, f)=o\{T(r, f)\}$ as $r \rightarrow \infty$ possibly outside a set of finite linear measure. We denote by $T(r)$ the maximum of $T(r, f)$ and $T(r, g)$. The notation $S(r)$ denotes any quantity satisfying $S(r)=o(T(r))$ as $r \longrightarrow \infty$, outside of a possible exceptional set of finite linear measure. 
A meromorphic function $a(z)$ is called a small function with respect to $f$, provided that $T(r, a)=S(r, f)$. The order of $f$ is defined by

$$
\rho(f)=\limsup _{r \longrightarrow \infty} \frac{\log T(r, f)}{\log r} .
$$

Definition 1.1. [7] Let $k$ be a nonnegative integer or infinity. For a $\in$ $\mathbb{C} \cup\{\infty\}$ we denote by $E_{k}(a ; f)$ the set of all a-points of $f$, where an a-point of multiplicity $m$ is counted $m$ times if $m \leq k$ and $k+1$ times if $m>k$. If $E_{k}(a ; f)=E_{k}(a ; g)$, we say that $f, g$ share the value a with weight $k$. We write $f, g$ share $(a, k)$ to mean that $f, g$ share the value a with weight $k$.

We now require the following definitions.

Definition 1.2. [6] For $a \in \mathbb{C} \cup\{\infty\}$ we denote by $N(r, a ; f \mid=1)$ the counting function of simple a points of $f$. For a positive integer $m$ we denote by $N(r, a ; f \mid \leq m)(N(r, a ; f \mid \geq m))$ the counting function of those a points of $f$ whose multiplicities are not greater(less) than $m$ where each a point is counted according to its multiplicity. $\bar{N}(r, a ; f \mid \leq m)(\bar{N}(r, a ; f \mid \geq m))$ are defined similarly, where in counting the a-points of $f$ we ignore the multiplicities. Also $N(r, a ; f \mid<m), N(r, a ; f \mid>m), \bar{N}(r, a ; f \mid<m)$ and $\bar{N}(r, a ; f \mid>m)$ are defined analogously.

Definition 1.3. [7] Let $k \in \mathbb{N} \cup\{\infty\}$. We denote by $N_{k}(r, a ; f)$ the counting function of a-points of $f$, where an a-point of multiplicity $m$ is counted $m$ times if $m \leq k$ and $k$ times if $m>k$. Then $N_{k}(r, a ; f)=\bar{N}(r, a ; f)+$ $\bar{N}(r, a ; f \mid \geq 2)+\ldots+\bar{N}(r, a ; f \mid \geq k)$. Clearly $N_{1}(r, a ; f)=\bar{N}(r, a ; f)$.

For the sake of simplicity we also use the notation

$$
m^{*}:= \begin{cases}m, & \text { if } m \leq k+1 \\ k+2, & \text { if } m>k+1\end{cases}
$$

where $m(\geq 1)$ and $k(\geq 0)$ are integers.

We first recall the following uniqueness result of X. G. Qi, L. Z. Yang and K. Liu [9] obtained in 2010.

Theorem A. [9] Let $f(z)$ and $g(z)$ be two transcendental entire functions of finite order and $\eta$ be a non-zero complex constant and let $n \geq 6$ be an integer. If $f^{n}(z) f(z+\eta)$ and $g^{n}(z) g(z+\eta)$ share $1 C M$, then either $f(z) g(z)=t_{1}$ or $f(z)=t_{2} g(z)$ for some constants $t_{1}$ and $t_{2}$ satisfying $t_{1}^{n+1}=t_{2}^{n+1}=1$.

Next we state Zhang's [12] following result. 
Theorem B. [12] Let $f(z)$ and $g(z)$ be two transcendental entire functions of finite order and $\alpha(z)(\not \equiv 0)$ be a small function with respect to both $f(z)$ and $g(z)$. Suppose that $\eta$ is a nonzero complex constant and $n \geq 7$ is an integer. If $f^{n}(z)(f(z)-1) f(z+\eta)$ and $g^{n}(z)(g(z)-1) g(z+\eta)$ share $\alpha(z)$ $C M$, then $f(z) \equiv g(z)$.

In 2013, S. S. Bhoosnurmath and S. R. Kabbur [1] improved Theorem B in the following manner.

Theorem C. Let $f(z)$ and $g(z)$ be two transcendental entire functions of finite order and $\alpha(z)(\equiv \equiv)$ be a small function with respect both $f(z)$ and $g(z)$. Suppose that $c$ is a nonzero complex constant and $n, m$ are positive integers such that $n \geq m+6$. If $f^{n}(z)\left(f^{m}(z)-1\right) f(z+c)$ and $g^{n}(z)\left(g^{m}(z)-1\right) g(z+c)$ share $\alpha(z) C M$, then $f(z) \equiv \operatorname{tg}(z)$, where $t^{m}=1$.

Recently generalizing Theorem C, P. Sahoo and B. Saha [8] proved the following results.

Theorem D. Let $f(z)$ and $g(z)$ be two transcendental entire functions of finite order and $\alpha(z)(\not \equiv 0)$ be a small function with respect both $f(z)$ and $g(z)$. Suppose that $c$ is a nonzero complex constant, $n(\geq 1), m(\geq 1)$ and $k(\geq 0)$ are integers satisfying $n \geq 2 k+m+6$. If $\left(f^{n}(z)\left(f^{m}(z)-1\right) f(z+c)\right)^{(k)}$ and $\left(g^{n}(z)\left(g^{m}(z)-1\right) g(z+c)\right)^{(k)}$ share $(\alpha(z), 2)$, then $f(z) \equiv \operatorname{tg}(z)$, where $t^{m}=1$.

Theorem E. Let $f(z)$ and $g(z)$ be two transcendental entire functions of finite order and $\alpha(z)(\not \equiv 0)$ be a small function with respect both $f(z)$ and $g(z)$. Suppose that $c$ is a nonzero complex constant, $n(\geq 1), m(\geq 1)$ and $k(\geq 0)$ are integers satisfying $n \geq 2 k+m+6$, when $m \leq k+1$ and $n \geq 4 k-m+10$, when $m>k+1$. If $\left(f^{n}(z)(f(z)-1)^{m} f(z+c)\right)^{(k)}$ and $\left(g^{n}(z)(g(z)-1)^{m} g(z+c)\right)^{(k)}$ share $(\alpha(z), 2)$, then either $f(z) \equiv g(z)$ or $f(z)$ and $g(z)$ satisfy the algebraic equation $R(f, g)=0$, where $R(f, g)$ is given by

$$
R\left(\omega_{1}, \omega_{2}\right)=\omega_{1}^{n}\left(\omega_{1}-1\right)^{m} \omega_{1}(z+c)-\omega_{2}^{n}\left(\omega_{2}-1\right)^{m} \omega_{2}(z+c) .
$$

The two theorems Theorem D [8] and Theorem E [8] stated above are no doubt a useful contribution in the field differential polynomial of shift operators. But unfortunately there are some gaps in the proof of theorems.

For example we consider page 41, 8-th line from top under the case $F G \equiv$ 1. The authors said

$$
N\left(r, \frac{1}{f}\right)=S(r, f), \quad N\left(r, \frac{1}{f-1}\right)=S(r, f) .
$$


But when

$$
\left[f^{n}\left(f^{m}-1\right) f(z+c)\right]^{(k)}\left[g^{n}\left(g^{m}-1\right) g(z+c)\right]^{(k)} \equiv \alpha^{2}(z),
$$

one can not always conclude $N\left(r, \frac{1}{f-1}\right)=S(r, f)$ under the situation. Actually $N\left(r, \frac{1}{f-1}\right)=S(r, f)$ is true only when zeros of $f-1$ are of multiplicities at least $k+1$.

Again we consider page 42 , 4 -th line from top under the case $F G \equiv 1$. The authors here also claimed

$$
N\left(r, \frac{1}{f}\right)=S(r, f), \quad N\left(r, \frac{1}{f-1}\right)=S(r, f) .
$$

But with $m \leq k, N\left(r, \frac{1}{f-1}\right)=S(r, f)$ is not always true under the situation when

$$
\left[f^{n}(z)(f(z)-1)^{m} f(z+c)\right]^{(k)}\left[g^{n}(z)(g(z)-1)^{m} g(z+c)\right]^{(k)} \equiv \alpha^{2}(z) .
$$

Actually here $N\left(r, \frac{1}{f-1}\right)=S(r, f)$ happens only when zeros of $f-1$ are of multiplicities at least $k+1$.

So the validity of the theorems $\mathrm{D}$ and $\mathrm{E}$ are at stake. So it will be interesting to find the correct form of the theorems. In the paper we rectify the errors in Theorems $\mathrm{D}$ and $\mathrm{E}$ at the cost of considering the fact that $\alpha(z)(\not \equiv 0)$ be a small function with respect to $f$ and $g$ with finitely many zeros which improve and generalize all the results demonstrated so far. We now present the following two theorems which are the main results of the paper.

Theorem 1.1. Let $f(z)$ and $g(z)$ be two transcendental entire functions of finite order, $c_{j}(j=1,2, \ldots, s)$ be finite complex constants and $\alpha(z)(\not \equiv 0)$ be a small function with respect both $f(z)$ and $g(z)$ with finitely many zeros. Suppose that $n(\geq 1), m(\geq 1)$ and $k(\geq 0)$ are integers satisfying $n \geq 2 k+$ $2 m^{*}-m+s+5$. If $\left(f^{n}(z)(f(z)-1)^{m} \prod_{j=1}^{s} f\left(z+c_{j}\right)\right)^{(k)}$ and $\left(g^{n}(z)(g(z)-\right.$ $\left.1)^{m} \prod_{j=1}^{s} g\left(z+c_{j}\right)\right)^{(k)}$ share $(\alpha(z), 2)$, then either $f(z) \equiv g(z)$ or $f(z)$ and $g(z)$ satisfy the equation $R(f, g)=0$, where $R(f, g)$ is given by $R\left(\omega_{1}, \omega_{2}\right)=$ $\omega_{1}^{n}\left(\omega_{1}-1\right)^{m} \prod_{j=1}^{s} \omega_{1}\left(z+c_{j}\right)-\omega_{2}^{n}\left(\omega_{2}-1\right)^{m} \prod_{j=1}^{s} \omega_{2}\left(z+c_{j}\right)$.

Theorem 1.2. Let $f(z)$ and $g(z)$ be two transcendental entire functions of finite order, $c_{j}(j=1,2, \ldots, s)$ be finite complex constants and $\alpha(z)(\not \equiv 0)$ be a small function with respect both $f(z)$ and $g(z)$ with finitely many zeros. Suppose that $n(\geq 1), m(\geq 1)$ and $k(\geq 0)$ are integers satisfying $n \geq$ $\max \{2 k+m+s+5,3 s+3\}$. If $\left(f^{n}(z)\left(f^{m}(z)-1\right) \prod_{j=1}^{s} f\left(z+c_{j}\right)\right)^{(k)}$ and $\left(g^{n}(z)\left(g^{m}(z)-1\right) \prod_{j=1}^{s} g\left(z+c_{j}\right)\right)^{(k)}$ share $(\alpha(z), 2)$, then $f(z) \equiv \operatorname{tg}(z)$ for some constant $t$ such that $t^{n+s}=t^{m}=1$. 
Remark 1.1. When $m>k+1$, then the above Theorem 1.1 holds without the condition " $\alpha(z)$ with finitely many zeros".

Definition 1.4. We denote by $N\left(r, \infty ; f^{n}(z) f(z+c)=f(z) \mid f(z+c) \neq \infty\right)$ the counting function of those common poles of $f^{n}(z) f(z+c)$ and $f(z)$ in $|z|<r$, where each such point is not a pole of $f(z+c)$ and each such point is counted according to its multiplicity in $N\left(r, \infty ; f^{n}(z) f(z+c)\right)$. We denote by $N(r, \infty ; f(z+c) \mid f(z)=\infty)$ the counting function of common poles of $f(z)$ and $f(z+c)$ in $|z|<r$, where each such point is counted according to its multiplicity in $N\left(r, \infty ; f^{n} f(z+c)\right)$ and we denote by $N\left(r, \infty ; f^{n}(z) f(z+\right.$ $c)=f(z+c) \mid f(z) \neq \infty)$ the counting function of those common poles of $f^{n}(z) f(z+c)$ and $f(z+c)$ in $|z|<r$, where each such point is not a pole of $f(z)$ and each such point is counted according to its multiplicity in $N\left(r, \infty ; f^{n}(z) f(z+c)\right)$.

\section{Lemmas}

Lemma 2.1. [10] Let $f$ be a non-constant meromorphic function and let $a_{n}(z)(\not \equiv 0), a_{n-1}(z), \ldots, a_{0}(z)$ be meromorphic functions such that $T\left(r, a_{i}(z)\right)=S(r, f)$ for $i=0,1,2, \ldots, n$. Then

$$
T\left(r, a_{n} f^{n}+a_{n-1} f^{n-1}+\ldots+a_{1} f+a_{0}\right)=n T(r, f)+S(r, f) .
$$

Lemma 2.2. [13] Let $f$ be a non-constant meromorphic function and $p, k$ be positive integers. Then

$$
\begin{gathered}
N_{p}\left(r, 0 ; f^{(k)}\right) \leq T\left(r, f^{(k)}\right)-T(r, f)+N_{p+k}(r, 0 ; f)+S(r, f), \\
N_{p}\left(r, 0 ; f^{(k)}\right) \leq k \bar{N}(r, \infty ; f)+N_{p+k}(r, 0 ; f)+S(r, f) .
\end{gathered}
$$

Lemma 2.3. [2] Let $f(z)$ be a meromorphic function of finite order $\rho$, and let $c \in \mathbb{C} \backslash\{0\}$ be fixed. Then for each $\varepsilon>0$, we have

$$
m\left(r, \frac{f(z+c)}{f(z)}\right)+m\left(r, \frac{f(z)}{f(z+c)}\right)=O\left(r^{\rho-1+\varepsilon}\right) .
$$

The following lemma has little modifications of the original version (Theorem 2.1 of $[2])$ 
Lemma 2.4. [4] Let $f$ be a non-constant meromorphic function of finite order and $c \in \mathbb{C}$. Then

$$
\begin{aligned}
& N(r, 0 ; f(z+c)) \leq N(r, 0 ; f(z))+S(r, f), \\
& N(r, \infty ; f(z+c)) \leq N(r, \infty ; f)+S(r, f), \\
& \bar{N}(r, 0 ; f(z+c)) \leq \bar{N}(r, 0 ; f(z))+S(r, f), \\
& \bar{N}(r, \infty ; f(z+c)) \leq \bar{N}(r, \infty ; f)+S(r, f) .
\end{aligned}
$$

Arguing a similar manner as in Lemma 2.6 [3] we obtain the following lemma.

Lemma 2.5. Let $f(z)$ be an entire function of finite order $\rho$ and $c_{j}(j=$ $1,2, \ldots, s)$ be finite complex constants. Let $m(\geq 0), n(\geq 1)$ be integers and $P(\omega)=a_{m} \omega^{m}+a_{m-1} \omega^{m-1}+\ldots+a_{1} \omega+a_{0}$ be a nonzero polynomial. Then for each $\varepsilon>0$, we have

$$
T\left(r, f^{n}(z) P(f)(z) \prod_{j=1}^{s} f\left(z+c_{j}\right)\right)=(n+m+s) T(r, f)+O\left(r^{\rho-1+\varepsilon}\right) .
$$

Lemma 2.6. Let $f(z)$ be a transcendental meromorphic function of finite order and and $c_{j}(j=1,2, \ldots, s)$ be finite complex constants. Suppose $n(\geq$ $1)$ is an integer such that $n>s$. Let $\Phi(z)=f^{n}(z) F_{s}(z)$, where $F_{s}(z)=$ $\prod_{j=1}^{s} f\left(z+c_{j}\right)$. Then we have

$$
(n-s) T(r, f) \leq T(r, \Phi)+S(r, f) .
$$

Proof. Note that

$$
\begin{aligned}
& N(r, \infty ; \Phi(z)) \\
= & N\left(r, \infty ; f^{n}(z)=f(z) \mid F_{s}(z) \neq \infty\right)+N\left(r, \infty ; F_{s}(z) \mid f(z)=\infty\right) \\
& +N\left(r, \infty ; f^{n}(z) F_{s}(z)=F_{s}(z) \mid f(z) \neq \infty\right) \\
\geq \quad & N\left(r, \infty ; f^{n}(z)\right)-N\left(r, 0 ; F_{s}(z)\right),
\end{aligned}
$$

i.e.,

$$
N\left(r, \infty ; f^{n}\right) \leq N(r, \infty ; \Phi)+N\left(r, 0 ; F_{s}(z)\right)+S(r, f)
$$


Now by Lemmas 2.3 and 2.4 we have

$$
\begin{aligned}
& m\left(r, f^{n}\right)=m\left(r, \frac{\Phi}{F_{s}(z)}\right) \\
\leq & m(r, \Phi)+m\left(r, \frac{1}{F_{s}(z)}\right)+S(r, f) \\
= & m(r, \Phi)+T\left(r, F_{s}(z)\right)-N\left(r, 0 ; F_{s}(z)\right)+S(r, f) \\
= & m(r, \Phi)+N\left(r, \infty ; F_{s}(z)\right)+m\left(r, F_{s}(z)\right)-N\left(r, 0 ; F_{s}(z)\right)+S(r, f) \\
\leq & m(r, \Phi)+N\left(r, \infty ; F_{s}(z)\right)+m\left(r, \frac{F_{s}(z)}{f^{s}(z)}\right)+m\left(r, f^{s}(z)\right)-N\left(r, 0 ; F_{s}(z)\right) \\
& +S(r, f) \\
= & m(r, \Phi)+s N(r, \infty ; f)+s m(r, f)-N\left(r, 0 ; F_{s}(z)\right)+S(r, f) \\
= & m(r, \Phi)+s T(r, f)-N\left(r, 0 ; F_{s}(z)\right)+S(r, f) .
\end{aligned}
$$

By Lemma 2.1 we get

$$
n T(r, f)=N\left(r, \infty ; f^{n}\right)+m\left(r, f^{n}\right) \leq T(r, \Phi)+s T(r, f)+S(r, f),
$$

i.e.,

$$
(n-s) T(r, f) \leq T(r, \Phi)+S(r, f) .
$$

This completes the Lemma.

Lemma 2.7. Let $f(z), g(z)$ be two transcendental entire functions of finite order and $c_{j}(j=1,2, \ldots, s)$ be finite complex constants. Let $m(\geq 1)$ and $n(\geq 1)$ be integers such that $n \geq 3 s+3$. If

$$
f^{n}(z)\left(f^{m}(z)-1\right) \prod_{j=1}^{s} f\left(z+c_{j}\right) \equiv g^{n}(z)\left(g^{m}(z)-1\right) \prod_{j=1}^{s} g\left(z+c_{j}\right),
$$

then $f(z) \equiv t g(z)$ for some constant $t$ such that $t^{m}=t^{n+s}=1$

Proof. Suppose

$$
f^{n}(z)\left(f^{m}(z)-1\right) \prod_{j=1}^{s} f\left(z+c_{j}\right) \equiv g^{n}(z)\left(g^{m}(z)-1\right) \prod_{j=1}^{s} g\left(z+c_{j}\right) .
$$

Let $h=\frac{f}{g}$. Clearly from (2.3) we get

$$
g^{m}(z)\left[h^{n+m}(z) H_{s}(z)-1\right] \equiv h^{n}(z) H_{s}(z)-1,
$$


where $H_{s}(z)=\prod_{j=1}^{s} h\left(z+c_{j}\right)$. First we suppose that $h$ is non-constant. We assert that both $h^{n+m}(z) H_{s}(z)$ and $h^{n}(z) H_{s}(z)$ are non-constant. If not, let $h^{n+m}(z) H_{s}(z) \equiv c_{1} \in \mathbb{C} \backslash\{0\}$. Then we have

$$
h^{n+m}(z) \equiv \frac{c_{1}}{H_{s}(z)} .
$$

Now by Lemmas 2.1, 2.3 and 2.4 we get

$$
\begin{aligned}
(n+m) T(r, h) & =T\left(r, h^{n+m}\right)+S(r, h) \\
& =T\left(r, \frac{c_{1}}{H_{s}(z)}\right)+S(r, h) \\
& \leq \sum_{j=1}^{s}\left[N\left(r, 0 ; h\left(z+c_{j}\right)\right)+m\left(r, \frac{1}{h\left(z+c_{j}\right)}\right)\right]+S(r, h) \\
& \leq \sum_{j=1}^{s} N(r, 0 ; h(z))+\sum_{j=1}^{s} m\left(r, \frac{1}{h(z)}\right)+S(r, h) \\
& \leq s T(r, h)+S(r, h),
\end{aligned}
$$

which is a contradiction. Similarly we can prove that $h^{n}(z) H_{s}(z)$ is nonconstant. Thus from (2.4) we have

$$
f^{m}(z) \equiv h^{m}(z) \frac{h^{n}(z) H_{s}(z)-1}{h^{n+m}(z) H_{s}(z)-1} \quad \text { and } \quad g^{m}(z) \equiv \frac{h^{n}(z) H_{s}(z)-1}{h^{n+m}(z) H_{s}(z)-1} .
$$

Let $z_{0}$ be a zero of $h^{n+m}(z) H_{s}(z)-1$. Since $g$ is an entire function, it follows that $z_{0}$ is also a zero of $h^{n}(z) H_{s}(z)-1$. Then clearly $h^{m}\left(z_{0}\right)-1=0$ and so

$$
\bar{N}\left(r, 1 ; h^{n+m} H_{s}(z)\right) \leq \bar{N}\left(r, 1 ; h^{m}\right) \leq m T(r, h)+O(1) .
$$

So in view of Lemmas 2.1, 2.4, 2.6 and the second fundamental theorem we get

$$
\begin{aligned}
& (n+m-s) T(r, h) \\
= & T\left(r, h^{n+m}(z) H_{s}(z)\right)+S(r, h) \\
\leq \quad & \bar{N}\left(r, 0 ; h^{n+m} H_{s}(z)\right)+\bar{N}\left(r, \infty ; h^{n+m} H_{s}(z)\right)+\bar{N}\left(r, 1 ; h^{n+m} H_{s}(z)\right) \\
& +S(r, h) \\
\leq & \bar{N}(r, 0 ; h)+\sum_{j=1}^{s}\left[\bar{N}\left(r, 0 ; h\left(z+c_{j}\right)\right)+\bar{N}\left(r, \infty ; h\left(z+c_{j}\right)\right)\right]+\bar{N}(r, \infty ; h) \\
& +m T(r, h)+S(r, h) \\
\leq \quad & N(r, 0 ; h)+\sum_{j=1}^{s}[N(r, 0 ; h(z))+N(r, \infty ; h(z))]+N(r, \infty ; h) \\
& +m T(r, h)+S(r, h) \\
\leq & (m+2 s+2) T(r, h)+S(r, h),
\end{aligned}
$$


which contradicts with $n>3 s+2$. Hence $h$ is a constant. Since $g$ is transcendental entire function, from (2.4) we have

$$
h^{n+m}(z) \prod_{j=1}^{s} h\left(z+c_{j}\right)-1 \equiv 0 \Longleftrightarrow h^{n}(z) \prod_{j=1}^{s} h\left(z+c_{j}\right)-1 \equiv 0
$$

and so $h^{m}(z)=1, h^{n+1}=1$. Thus $f(z) \equiv \operatorname{tg}(z)$ for a constant $t$ such that $t^{m}=t^{n+s}=1$.

This completes the the proof.

Remark 2.1. Clearly Lemma 2.7 rectifies, improves and generalizes Lemma 5 [1].

Lemma 2.8. [7] Let $f$ and $g$ be two non-constant meromorphic functions sharing $(1,2)$. Then one of the following holds:

(i) $T(r, f) \leq N_{2}(r, 0 ; f)+N_{2}(r, 0 ; g)+N_{2}(r, \infty ; f)+N_{2}(r, \infty ; g)+S(r, f)+$ $S(r, g)$,

(ii) $f g \equiv 1$,

(iii) $f \equiv g$.

Lemma 2.9. Let $f(z), g(z)$ be two transcendental entire functions of finite order and $c_{j}(j=1,2, \ldots, s)$ be finite complex constants. Let $k(\geq 1), m(\geq 0)$, $n(\geq 1)$ be integers such that $n>k$. Suppose $P_{1}(\omega)=a_{m} \omega^{m}+a_{m-1} \omega^{m-1}+$ $\ldots+a_{1} \omega+a_{0}$ is a nonzero polynomial. Let $a(z)(\not \equiv 0, \infty)$ be a small function with respect to $f$ and $g$ with finitely many zeros. If

$$
\left[f^{n}(z) P_{1}(f)(z) \prod_{j=1}^{s} f\left(z+c_{j}\right)\right]^{(k)}\left[g^{n}(z) P_{1}(g)(z) \prod_{j=1}^{s} g\left(z+c_{j}\right)\right]^{(k)} \equiv a^{2}(z)
$$

then $P_{1}(\omega)$ reduces to a nonzero monomial, namely $P_{1}(\omega)=a_{i} \omega^{i} \not \equiv 0$ for some $i \in\{0,1, \ldots, m\}$.

Proof. Suppose on the contrary $P_{1}(\omega)$ does not reduce to a nonzero monomial, then, without loss of generality, we assume that $P_{1}(\omega)=a_{m} \omega^{m}+$ $a_{m-1} \omega^{m-1}+\ldots+a_{1} \omega+a_{0}$, where $a_{0} \neq 0, a_{1}, \ldots, a_{m-1}, a_{m} \neq 0$ are complex constants.

Since the number of zeros of $a(z)$ is finite, it follows that $f$ as well as $g$ has finitely many zeros. Then $f(z)$ takes the form

$$
f(z)=h(z) e^{\alpha(z)}
$$


where $h$ is a nonzero polynomial and $\alpha$ is a non-constant polynomial. Let

$$
h_{i}(z)=h^{n+i}(z) \prod_{j=1}^{s} h\left(z+c_{j}\right) \text { and } \alpha_{i}(z)=(n+i) \alpha(z)+\sum_{j=1}^{s} \alpha\left(z+c_{j}\right) \text {, }
$$

where $i=0,1,2, \ldots, m$. Clearly

$$
f^{n+i}(z) \prod_{j=1}^{s} f\left(z+c_{j}\right)=h_{i}(z) e^{\alpha_{i}(z)}
$$

where $i=0,1,2, \ldots, m$. Then by induction we have

$$
\left[a_{i} f^{n+i}(z) \prod_{j=1}^{s} f\left(z+c_{j}\right)\right]^{(k)}=t_{i}\left(\alpha_{i}^{\prime}, \alpha_{i}^{\prime \prime}, \ldots, \alpha_{i}^{(k)}, h_{i}, h_{i}^{\prime}, \ldots, h_{i}^{(k)}\right) e^{\alpha_{i}}
$$

where $t_{i}\left(\alpha_{i}^{\prime}, \alpha_{i}^{\prime \prime}, \ldots, \alpha_{i}^{(k)}, h_{i}, h_{i}^{\prime}, \ldots, h_{i}^{(k)}\right)(i=0,1,2, \ldots, m)$ are differential polynomials in

$\alpha_{i}^{\prime}, \alpha_{i}^{\prime \prime}, \ldots, \alpha_{i}^{(k)}, h_{i}, h_{i}^{\prime}, \ldots, h_{i}^{(k)}$. Since $f(z)$ is a transcendental entire function, from (2.7) we see that

$$
t_{i}\left(\alpha_{i}^{\prime}, \alpha_{i}^{\prime \prime}, \ldots, \alpha_{i}^{(k)}, h_{i}, h_{i}^{\prime}, \ldots, h_{i}^{(k)}\right) \not \equiv 0,
$$

for $i=0,1,2, \ldots, m$. Note that

$$
\begin{aligned}
{\left[f^{n}(z) P_{1}(f)(z) \prod_{j=1}^{s} f\left(z+c_{j}\right)\right]^{(k)} } & =\sum_{i=0}^{m}\left[a_{i} f^{n+i}(z) \prod_{j=1}^{s} f\left(z+c_{j}\right)\right]^{(k)} \\
& =\sum_{i=0}^{m} t_{i}(z) e^{\alpha_{i}(z)} \\
& =e^{n \alpha(z)+\sum_{j=1}^{s} \alpha\left(z+c_{j}\right)} \sum_{i=0}^{m} t_{i}(z) e^{i \alpha(z)}
\end{aligned}
$$

and so $\left[f^{n} P_{1}(f) \prod_{j=1}^{s} f\left(z+c_{j}\right)\right]^{(k)} \not \equiv 0$. Note that $h_{i}(z)$ and $\alpha_{i}(z)$ are polynomials, where $i=0,1, \ldots, m$. Consequently each $t_{i}(z)(i=0,1, \ldots, m)$ are also polynomials. Since $f(z)$ is a transcendental entire function, it follows that $T\left(r, t_{i}\right)=S(r, f)$ for $i=0,1,2, \ldots, m$. Note that

$$
\bar{N}\left(r, 0 ;\left[f^{n} P_{1}(f) \prod_{j=1}^{s} f\left(z+c_{j}\right)\right]^{(k)}\right) \leq N\left(r, 0 ; \alpha^{2}(z)\right) \leq S(r, f) .
$$

Now from (2.8) we have

$$
\bar{N}\left(r, 0 ; t_{m} e^{m \alpha(z)}+\ldots+t_{1} e^{\alpha(z)}+t_{0}\right) \leq S(r, f) .
$$


Since $t_{m} e^{m \alpha(z)}+\ldots+t_{1} e^{\alpha(z)}$ is a transcendental entire function and $t_{0}(z)$ is a polynomial, it follows that $t_{0}$ is a small function of $t_{m} e^{m \alpha(z)}+\ldots+t_{1} e^{\alpha(z)}$. So from (2.9) and using second fundamental theorem for small functions (see [11]), we obtain

$$
\begin{aligned}
& m T(r, f) \\
= & T\left(r, t_{m} e^{m \alpha}+\ldots+t_{1} e^{\alpha}\right)+S(r, f) \\
\leq & \bar{N}\left(r, 0 ; t_{m} e^{m \alpha}+\ldots+t_{1} e^{\alpha}\right)+\bar{N}\left(r, 0 ; t_{m} e^{m \alpha}+\ldots+t_{1} e^{\alpha}+t_{0}\right)+S(r, f) \\
\leq & \bar{N}\left(r, 0 ; t_{m} e^{(m-1) \alpha}+\ldots+t_{1}\right)+S(r, f) \\
\leq & (m-1) T(r, f)+S(r, f),
\end{aligned}
$$

which is a contradiction. Hence $P_{1}(\omega)$ is reduced to a nonzero monomial, namely $P_{1}(\omega)=a_{i} \omega^{i} \not \equiv 0$ for some $i \in\{0,1, \ldots m\}$. This completes the proof of the lemma.

Remark 2.2. If $P_{1}(\omega)=a_{m} \omega^{m}+a_{m-1} \omega^{m-1}+\ldots+a_{1} \omega+a_{0}$ be a polynomial, where $a_{0} \neq 0, a_{1}, \ldots, a_{m} \neq 0$ are complex constants, then by Lemma 2.9 we have

$$
\left[f^{n}(z) P_{1}(f)(z) \prod_{j=1}^{s} f\left(z+c_{j}\right)\right]^{(k)}\left[g^{n}(z) P_{1}(g)(z) \prod_{j=1}^{s} g\left(z+c_{j}\right)\right]^{(k)} \not \equiv a^{2}(z) .
$$

Lemma 2.10. Let $f(z)$ and $g(z)$ be two transcendental entire functions of finite order, $c_{j}(j=1,2, \ldots, s)$ be finite complex constants. Suppose that $n(\geq 1), m(\geq 1)$ and $k(\geq 0)$ are integers satisfying $n \geq 2 k+2 m^{*}-m+s+3$. If $\left(f^{n}(z)(f(z)-1)^{m} \prod_{j=1}^{s} f\left(z+c_{j}\right)\right)^{(k)} \equiv\left(g^{n}(z)(g(z)-1)^{m} \prod_{j=1}^{s} g\left(z+c_{j}\right)\right)^{(k)}$, then $f^{n}(z)(f(z)-1)^{m} \prod_{j=1}^{s} f\left(z+c_{j}\right) \equiv g^{n}(z)(g(z)-1)^{m} \prod_{j=1}^{s} g\left(z+c_{j}\right)$.

Proof. Proof of Lemma follows from the proof of Theorem 3 [8].

Lemma 2.11. Let $f(z)$ and $g(z)$ be two transcendental entire functions of finite order, $c_{j}(j=1,2, \ldots, s)$ be finite complex constants. Suppose that $n(\geq 1), m(\geq 1)$ and $k(\geq 0)$ are integers satisfying $n \geq 2 k+m+s+3$. If $\left(f^{n}(z)\left(f^{m}(z)-1\right) \prod_{j=1}^{s} f\left(z+c_{j}\right)\right)^{(k)} \equiv\left(g^{n}(z)\left(g^{m}(z)-1\right) \prod_{j=1}^{s} g\left(z+c_{j}\right)\right)^{(k)}$, then $f^{n}(z)\left(f^{m}(z)-1\right) \prod_{j=1}^{s} f\left(z+c_{j}\right) \equiv g^{n}(z)\left(g^{m}(z)-1\right) \prod_{j=1}^{s} g\left(z+c_{j}\right)$.

Proof. Proof of Lemma follows from Theorem 3 [8].

\section{Proofs of the Theorems}

Proof of Theorem 1.1. Let

$$
F(z)=\left[f^{n}(z) P(f)(z) \overline{F_{s}}(z)\right]^{(k)}, \quad G(z)=\left[g^{n}(z) P(g)(z) \overline{G_{s}}(z)\right]^{(k)},
$$


where $\bar{F}_{s}(z)=\prod_{j=1}^{s} f\left(z+c_{j}\right), \bar{G}_{s}(z)=\prod_{j=1}^{s} g\left(z+c_{j}\right)$ and $P(\omega)=(\omega-1)^{m}$. Also we define $F_{1}(z)=\frac{F(z)}{\alpha(z)}$ and $G_{1}(z)=\frac{G(z)}{\alpha(z)}$. Then $F_{1}$ and $G_{1}$ share $(1,2)$ except for the zeros and poles of $\alpha(z)$. Now applying Lemma 2.8 we see that one of the following three cases holds.

Case 1. Suppose

$$
\begin{aligned}
T\left(r, F_{1}\right) \leq & N_{2}\left(r, 0 ; F_{1}\right)+N_{2}\left(r, 0 ; G_{1}\right)+N_{2}\left(r, \infty ; F_{1}\right)+N_{2}\left(r, \infty ; G_{1}\right) \\
& +S\left(r, F_{1}\right)+S\left(r, G_{1}\right) .
\end{aligned}
$$

Using Lemmas 2.1, 2.2, 2.4 and 2.5 we get from the second fundamental theorem that

$$
\begin{aligned}
& (n+m+s) T(r, f) \\
\leq & T\left(r, f^{n}(z) P(f) \bar{F}_{s}\right) \\
\leq & T(r, F)+N_{k+2}\left(r, 0 ; f^{n} P(f) \bar{F}_{s}\right)-N_{2}(r, 0 ; F)+S(r, f) \\
\leq & T\left(r, F_{1}\right)+N_{k+2}\left(r, 0 ; f^{n} P(f) \bar{F}_{s}\right)-N_{2}(r, 0 ; F)+S(r, f) \\
\leq & N_{2}\left(r, 0 ; F_{1}\right)+N_{2}\left(r, 0 ; G_{1}\right)+N_{k+2}\left(r, 0 ; f^{n} P(f) \bar{F}_{s}\right)-N_{2}(r, 0 ; F) \\
& +S(r, f)+S(r, g) \\
\leq & N_{2}(r, 0 ; F)+N_{2}(r, 0 ; G)+N_{k+2}\left(r, 0 ; f^{n} P(f) \bar{F}_{s}\right)-N_{2}(r, 0 ; F) \\
& +S(r, f)+S(r, g) \\
\leq & N_{k+2}\left(r, 0 ; f^{n} P(f) \bar{F}_{s}\right)+N_{k+2}\left(r, 0 ; g^{n} P(g) \bar{G}_{s}\right)+S(r, f)+S(r, g) \\
\leq & N_{k+2}\left(r, 0 ; f^{n}\right)+N_{k+2}(r, 0 ; P(f))+N_{k+2}\left(r, 0 ; \bar{F}_{s}\right)+N_{k+2}\left(r, 0 ; g^{n}\right) \\
& +N_{k+2}(r, 0 ; P(g))+N_{k+2}\left(r, 0 ; \bar{G}_{s}\right)+S(r, f)+S(r, g) \\
\leq & (k+2) \bar{N}(r, 0 ; f)+m^{*} N(r, 0 ; f)+N\left(r, 0 ; \bar{F}_{s}\right)+(k+2) \bar{N}(r, 0 ; g) \\
& +m^{*} N(r, 0 ; g)+N\left(r, 0 ; \bar{G}_{s}\right)+S(r, f)+S(r, g) \\
\leq & \left(k+s+2+m^{*}\right) T(r, f)+\left(k+s+2+m^{*}\right) T(r, g)+S(r, f)+S(r, g) \\
\leq & \left(2 k+2 s+4+2 m^{*}\right) T(r)+S(r) .
\end{aligned}
$$

In a similar way we can obtain

$$
(n+m+s) T(r, g) \leq\left(2 k+2 s+4+2 m^{*}\right) T(r)+S(r) .
$$

Combining (3.1) and (3.2) we see that

$$
(n+m+s) T(r) \leq\left(2 k+2 s+4+2 m^{*}\right) T(r)+S(r),
$$

i.e

$$
\left(n+m-2 k-s-4-2 m^{*}\right) T(r) \leq S(r) .
$$


Since $n \geq 2 k+2 m^{*}-m+s+5$, (3.3) leads to a contradiction.

Case 2. Let $F_{1} \equiv G_{1}$. Then

$$
\left[f^{n}(z) P(f)(z) \prod_{j=1}^{s} f\left(z+c_{j}\right)\right]^{(k)} \equiv\left[g^{n}(z) P(g)(z) \prod_{j=1}^{s} g\left(z+c_{j}\right)\right]^{(k)} .
$$

Now by Lemma 2.10, we get

$$
f^{n}(z)(f(z)-1)^{m} \prod_{j=1}^{s} f\left(z+c_{j}\right) \equiv g^{n}(z)(g(z)-1)^{m} \prod_{j=1}^{s} g\left(z+c_{j}\right) .
$$

Let $h=\frac{f}{g}$. First we suppose that $h$ is non-constant. Then from (3.4) we can say that

$$
f^{n}(z)(f(z)-1)^{m} \prod_{j=1}^{s} f\left(z+c_{j}\right) \equiv g^{n}(z)(g(z)-1)^{m} \prod_{j=1}^{s} g\left(z+c_{j}\right),
$$

i.e., $f(z)$ and $g(z)$ satisfy the algebraic equation $R(f, g)=0$, where $R(f, g)$ is given by $R\left(\omega_{1}, \omega_{2}\right)=\omega_{1}^{n}\left(\omega_{1}-1\right)^{m} \prod_{j=1}^{s} \omega_{1}\left(z+c_{j}\right)-\omega_{2}^{n}\left(\omega_{2}-1\right)^{m} \prod_{j=1}^{s} \omega_{2}\left(z+c_{j}\right)$. Next we suppose that $h$ is a constant. Then from (3.4) we get

$$
\begin{aligned}
& f^{n}(z) \prod_{j=1}^{s} f\left(z+c_{j}\right) \sum_{i=0}^{m}(-1)^{i}{ }^{m} C_{m-i} f^{m-i}(z) \\
\equiv & g^{n}(z) \prod_{j=1}^{s} g\left(z+c_{j}\right) \sum_{i=0}^{m}(-1)^{i}{ }^{m} C_{m-i} g^{m-i}(z) .
\end{aligned}
$$

Now substituting $f=g h$ in $(3.5)$ we get

$$
\sum_{i=0}^{m}(-1)^{i m} C_{m-i} g^{m-i}(z)\left(h^{n+m+s-i}(z)-1\right) \equiv 0,
$$

which implies that $h=1$. Hence $f(z) \equiv g(z)$.

Case $3 . F_{1} G_{1} \equiv 1$. Then

$$
\begin{array}{r}
{\left[f^{n}(z)(f(z)-1)^{m} \prod_{j=1}^{s} f\left(z+c_{j}\right)\right]^{(k)}\left[g^{n}(z)(g(z)-1)^{m} \prod_{j=1}^{s} g\left(z+c_{j}\right)\right]^{(k)}} \\
\equiv \alpha^{2}(z) .
\end{array}
$$

Remaining part follows from Remark 2.2. This completes the proof. 
Proof of Theorem 1.2. Let

$$
\begin{aligned}
& F(z)=\left[f^{n}(z) P(f)(z) \prod_{j=1}^{s} f\left(z+c_{j}\right)\right]^{(k)}, \\
& G(z)=\left[g^{n}(z) P(g)(z) \prod_{j=1}^{s} g\left(z+c_{j}\right)\right]^{(k)},
\end{aligned}
$$

where $P(\omega)=\omega^{m}-1$. Also we define $F_{1}(z)=\frac{F(z)}{\alpha(z)}$ and $G_{1}(z)=\frac{G(z)}{\alpha(z)}$. Then $F_{1}$ and $G_{1}$ share $(1,2)$ except for the zeros and poles of $\alpha(z)$. Now applying Lemma 2.8 we see that one of the following three cases holds.

Case 1. Suppose

$$
\begin{aligned}
T\left(r, F_{1}\right) \leq & N_{2}\left(r, 0 ; F_{1}\right)+N_{2}\left(r, 0 ; G_{1}\right)+N_{2}\left(r, \infty ; F_{1}\right)+N_{2}\left(r, \infty ; G_{1}\right) \\
& +S\left(r, F_{1}\right)+S\left(r, G_{1}\right) .
\end{aligned}
$$

Now applying the same technique as in the proof of Theorem 1.1, we get

$$
(n-2 k-s-4-m) T(r) \leq S(r) \text {. }
$$

Since $n \geq 2 k+m+s+5$, we arrive at a contradiction.

Case 2. Let $F_{1} \equiv G_{1}$. Remaining part follows from Lemmas 2.7 and 2.11. Case 3. $F_{1} G_{1} \equiv 1$. Remaining part follows from Remark 2.2. This completes the proof.

\section{Acknowledgement}

The author wish to thank the referee for his/her valuable comments and suggestions towards the improvement of the paper.

\section{References}

[1] S. S. Bhoosnurmath and S. R. Kabbur, Value distribution and uniqueness theorems for difference of entire and meromorphic function, Int. J. Anal. Appl., $\mathbf{2}$, ( 2013), 124-136.

[2] Y. M. Chiang and S. J. Feng, On the Nevanlinna characteristic of $f(z+\eta)$ and difference equations in complex plane, Ramanujan J., 16, ( 2008), 105-129.

[3] M. R. Chen and Z. X. Chen, Properties of difference polynomials of entire functions with finite order, Chinese Annals of Mathematics, 33A, ( 2012), 359-374. 
[4] J. Heittokangas, R. Korhonen, I. Laine, J. Rieppo, and J. L. Zhang, Value sharing results for shifts of meromorphic function, and sufficient conditions for periodicity, J. Math. Anal. Appl., 355, (2009), 352-363.

[5] W. K. Hayman, Meromorphic Functions, The Clarendon Press, Oxford, 1964

[6] Value distribution of certain differential polynomials, Int. J. Math. Math. Sc., 28, ( 2001), 83-91.

[7] I. Lahiri, Weighted value sharing and uniqueness of meromorphic functions, Complex Var. Theory Appl., 46, (2001), 241-253.

[8] P. Sahoo and B. Saha, Value distribution and uniqueness of certain type of difference polynomials, Appl. Math. E-Notes, 16, (2016), 33-44.

[9] X. G. Qi, L. Z. Yang, and K. Liu, Uniqueness and periodicity of meromorphic functions concerning the difference operator, Comput. Math. Appl., 60, ( 2010)), $1739-1746$.

[10] C. C. Yang, On deficiencies of differential polynomials II, Math. Z., 125, (1972), 107-112.

[11] K. Yamanoi, The second main theorem for small functions and related problems, Acta Math., 192, (2004), 225-294

[12] J. L. Zhang, Value distribution and shared sets of differences of meromorphic functions, J. Math. Anal. Appl., 367, (2010), 401-408.

[13] Q. C. Zhang, Meromorphic function that shares one small function with its derivative, J. Inequal. Pure Appl. Math., 6 (4), ( 2005), Art.116 [ ONLINE http://jipam.vu.edu.au/

Sujoy Majumder

Department of Mathematics,

Raiganj University,

Raiganj,

West Bengal, 733134,

India

E-mail: sujoy.katwa@gmail.com, smajumder05@yahoo.in

Received: 16.10 .2016

Accepted: 9.01.2017 